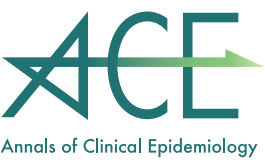

\title{
Long-term Functional Outcomes, Quality of Life, and Patient Trajectory in Trauma Survivors: A Study Protocol
}

\author{
Asuka Tsuchiya ${ }^{1,2}$, Yusuke Tsutsumi ${ }^{1,3}$, Hideo Yasunaga ${ }^{4}$, Susumu Yasuda ${ }^{1}, K^{2}$ ji Yuzawa ${ }^{1}$, \\ Shigeki Kushimoto ${ }^{5}$
}

\begin{abstract}
\section{BACKGROUND}

Trauma is a leading cause of the loss of social life and a major contributor to the global burden of disease. Although trauma-related short-term mortality has decreased worldwide, knowledge on long-term outcomes, including health-related quality of life, patient trajectory, and reintegration into society, is lacking.

OBJECTIVE

To create a comprehensive long-term trauma outcome database, describe patients' long-term outcomes in the first 2 years of injury, and explore the association between patients' background and long-term outcomes.

METHODS

This study will be a nationwide prospective cohort study. We will prospectively collect data on patients aged $\geq 16$ years with moderate to severe trauma (injury severity score $>12$ ) who are admitted to acute care facilities and discharged alive. After obtaining informed consent, we will follow the patients for 2 years and obtain data on their comprehensive long-term outcomes and social backgrounds. Thereafter, we will combine these new data with the existing data from two other databases (the Japan Trauma Data Bank and Diagnosis Procedure Combination database) and subsequently create a larger more comprehensive trauma database. ANALYSIS

We will focus on epidemiological and descriptive analyses and analyze the associations between patients' social backgrounds and long-term outcomes. A generalized linear mixedeffect logistic regression model and a random intercept per hospital will be used to adjust for baseline confounders and institutional differences.

CONTRIBUTION AND SIGNIFICANCE TO THE FIELD

This study will be an essential piece of evidence from a public health policy perspective and will recommend medical care optimized for each patient to help trauma survivors regain control over their lives.
\end{abstract}

KEY WORDS

patient-reported outcomes, trauma, long-term outcomes, trajectory, quality of life

\footnotetext{
1 Department of Emergency and Critical Care Medicine, National Hospital Organization Mito Medical Center

${ }^{2}$ Department of Health Service Research, Graduate School of Medicine, The University of Tokyo

${ }^{3}$ Department of Healthcare Epidemiology, Graduate School of Public Health and Medicine, Kyoto University

${ }^{4}$ Department of Clinical Epidemiology \& Health Economics, School of Public Health, The University of Tokyo

${ }^{5}$ Division of Emergency and Critical Care Medicine, Tohoku University Graduate School of Medicine

Corresponding author: Asuka Tsuchiya Department of Emergency and Critical Care Medicine, National Hospital Organization Mito Medical Center; Postal address, 280 Sakuranosato, Ibarakimachi, Higashiibarakigun, Ibaraki 3113193, Japan E-mail: asuka-t@umin.ac.jp

Received: October 15, 2020 Accepted: January 9, 2021

No.21-08

(C) 2021 Society for Clinical Epidemiology
} 


\section{INTRODUCTION}

$\mathrm{T}$ rauma is a serious global health problem that disrupts social life and accounts for more disability-adjusted life-years than any other disease [1-3]. Advances in treatment and improvements in trauma care facilities and systems have significantly reduced trauma-related short-term mortality in highincome countries. In Japan, approximately 95\% trauma patients were discharged alive in 2018 [4].

With an increase in survival rates, patients may experience the consequences of their injuries, such as persistent pain, limited movement, and sustained anxiety or depression, for extended periods $[5,6]$. This long-term sequela may prevent survivors from reintegrating into society, potentially resulting in other significant health problems $[7,8]$. In previous long-term follow-up studies, more than half of patients with trauma had residual physical limitations, and most patients required assistance with at least one activity of daily living. A previous study reported that quality of life domains, such as general health, physical functioning, physical pain, social roles, and social functioning, were significantly lower than the US norms 12 months after an injury [5]. In another study, the pain and discomfort that had resolved previously, worsened again 24 months later [6]. Furthermore, a study reported that $70 \%$ patients who were previously working or studying returned to their work or study 2 years after the injury [7]. Age, compensable status, and education levels were significantly associated with the long-term prognosis, and there was a cause-and-effect relationship between socioeconomic disadvantage and most outcomes [6].

Except a few areas, the health-related quality of life (HRQL) and patient trajectory or the proportion of patients reintegrating into society after survival from moderate to severe injury is poorly understood [9-11]. Although we have gradually recognized that social support systems are potentially at the core of recovery and we have focused more on long-term outcomes rather than traditional measures of injury severity, the existing trauma registries have not collected data on patients' social background or long-term outcomes $[5,6]$. To provide timely medical care, disability services, and adequate information to patients facing these sequelae, we must be aware of the comprehensive long-term outcomes and the social background of patients with trauma $[5,12]$.

Thus, the purpose of this study is to create a comprehensive long-term trauma outcome database, describe patients' long-term outcomes in the first 2 years of injury, and explore the association between patients' backgrounds and long-term outcomes.

\section{METHODS}

\section{STUDY DESIGN AND RESEARCH ETHICS APPROVAL}

This study will be a nationwide prospective cohort study. The Institutional Review Board of the National Hospital Organization Mito Medical Center and the Research Ethics Committee of the Japanese Association for Surgery of Trauma have approved this study. Informed consent will be obtained from each patient for using patients' personal information during follow-up.

\section{STUDY SETTING AND PARTICIPANTS}

Data will be collected from tertiary-level hospitals across Japan. These hospitals are similar to level 1 trauma centers in Europe and the United States and are certified by the Ministry of Health, Labour and Welfare. They can provide 24 -h specialty care in areas such as general surgery, cardiovascular surgery, orthopedic surgery, neurosurgery, anesthesiology, emergency medicine, radiology, internal medicine, and critical care [13].

\section{ELIGIBILITY CRITERIA}

Inclusion Criteria

The inclusion criteria will include the following: age $\geq 16$ years, direct transport from the scene of injury via ambulance or physician-staffed helicopter or ambulance, transferred to the hospital within $24 \mathrm{~h}$ of injury for advanced treatment, and injury severity score $>12$.

We will only include patients aged $\geq 16$ years as treatment procedures and drug dosages will not differ significantly among patients aged $\geq 16$ years in trauma care settings [14]. Moreover, existing validation studies on the population norm of the health-related questionnaires include people aged $\geq 16$ years [15-18].

\section{Exclusion Criteria}

The exclusion criteria will include the following: cardiopulmonary arrest on admission to the emergency department, patients with burns, and patient limitations that may prohibit long-term follow-up depending on the attending physician's judgment.

Patients with cardiopulmonary arrest have poor shortterm and long-term prognoses. Burns differ from other blunt or penetrating injuries as they are not accompanied by bleeding or obstructive shock, which can cause immediate death. In addition, the problems in patients with burns are different from those in patients with blunt or penetrating injuries [14]. 


\section{DATA COLLECTION}

\section{Sample Size and Recruitment}

This study will be observational and focused on epidemiological and descriptive analyses. We will additionally analyze the association between patients' social backgrounds and long-term outcomes, thereby omitting the need for a sample size calculation. However, we will set a reality-based target sample size of 3,600 patients for this study. Assuming that the target trauma severity is injury severity score $>12$, the average number of eligible patients per year for the 15 institutions that are members of the Japanese Society of Trauma Multicenter Clinical Research Committee is 100-120. Presuming that $80 \%$ of these patients would consent to participate in the study, the number of potential patients would be approximately 100. The participation of 15-20 institutions would be expected. This would result in 3,000 patients if 15 institutions participate and 4,000 patients if 20 institutions participate during the 2-year case registration period. Therefore, we adopted a middle value and set the number of cases at 3600. All patients eligible for this study will provide informed consent on admission, following which we will administer questionnaires to these patients.

\section{Collection of Data}

We will collect new data (patients' social background and comprehensive long-term outcomes) and combine them with data from two other databases-(i) the Japan Trauma Data Bank (JTDB) [19], a national trauma cohort database, and (ii) the Diagnosis Procedure Combination (DPC) database [20], a national administrative claims and discharge abstract database. Subse- quently, we will create a more comprehensive trauma database. A flow diagram of this study is shown in Fig. 1. Collection of Data on Patients' Social Background

The following data on patients' social backgrounds will collected: socioeconomic status, annual personal and household income, insurance status and insurer number, marital status, family configuration, place of residence, occupation, type of employment, level of education, presence of pre-injury disability, mental health status, presence of a family doctor, and the number of medications taken.

Collection of Data on Patients' Comprehensive Long-Term Outcomes

We will collect data on both qualitative and quantitative outcomes. Qualitative outcomes will include HRQL (EuroQol-5 dimensions-5 level, EQ-5D-5L; 12-Item Short-Form Health Survey version 2, SF-12v2), return to work-related outcomes, and place of residence. Quantitative outcomes will include long-term mortality, frequency of outpatient department visits, and frequency of readmissions.

i) HRQL

We will use the SF-12v2 and/or EQ-5D-5L. The EQ-5D-5L is a standardized and validated measure of health status developed by the EuroQol Group to provide a simple, generic measure of health for clinical and economic appraisal [21-23]. EQ-5D-5L instruments have been developed to describe and evaluate health across various disease areas, including trauma. The EQ-5D-5L consists of a descriptive component that gathers information on five domains (mobility, self-care, usual activities,




pain or discomfort, and anxiety or depression) and a visual analog scale that respondents use to rate their health from 0 (worst imaginable health state) to 100 (best imaginable health state) [18]. The Japanese language version and Japanese population norms are available [16].

The SF-12v2 includes 12 questions on functional health and well-being and provides physical and mental component summary scores based on psychometric analysis [15]. It is a practical, reliable, and validated measure of physical and mental health. The Japanese language version and Japanese population norms are available [17, 18]. The SF-12v2 and EQ-5D-5L also measure health status; however, the domains differ from each other. Thus, we will use both the HRQL questionnaires.

The paper version of the HRQL questionnaires will be mailed to the patients. In addition, we will be able to administer the questionnaire and request that the patients complete the questionnaire during face-to-face interviews. However, caregivers will be allowed to answer the questionnaire in cases of trauma patients with brain injury or dementia who are unable to answer. This will only be allowed for the EQ-5D-5L as the SF-12v2 cannot record answers by anyone other than the patient. Thus, we administer the EQ-5D-5L and the SF-12v2 if the patient is in a good state of consciousness at discharge from hospital. However, if the patient is in a poor state of consciousness, only the EQ-5D-5L will be administered to the caregiver (family member). The HRQL questionnaires will be mailed to the patient's current residence, such as their home, hospital, nursing home, long-term care facility, or residence with health and welfare services for elderly individuals, from the data management center. Each patient will be expected to complete the questionnaire and return it to the center. If no response is obtained for 2 weeks, the data center will resend the questionnaires. If no response is obtained again, the researcher will attempt to contact the patient via telephone and conduct a telephonic interview.

ii) Regarding all data, return to work-related outcomes, all-cause readmission rates, and outpatient department visitation rates, we will mail a special questionnaire to the patient's current residence together with the HRQL questionnaires and obtain them by return mail.

\section{JTDB}

The JTDB is the largest repository of national trauma data in Japan. Data were collected from 280 participating hospitals (227 tertiary-level and 53 secondary-level emergency hospitals). In 2018, approximately 78.3\% (227/290) of tertiary-level emergency hospitals in Japan participated [24], and approximately $93 \%$ of the data were collec- ted from these hospitals [14]. Although the JTDB is a voluntary registration system, it is strongly recommended that tertiary-level emergency hospitals and facilities that treat severe trauma register themselves. Registration is a requirement for trauma specialist facilities. The JTDB was initiated in 2003 and was revised in 2019 by the Japanese Association for Trauma Surgery (Trauma Registry Committee) and the Japanese Association for Acute Medicine (Committee for Clinical Care Evaluation). The Association for Japan Trauma Care Research plays a leading role in the training of the Abbreviated Injury Scale (AIS)-certified trauma registry coders. Data are prospectively and continuously recorded through a webbased format, and data are compiled in a data server at the Association for Japan Trauma Care Research. The Association for Japan Trauma Care Research analyzes the data and publishes an annual report [24].

The database contains the following information: patient's demographic data from pre-hospital to inhospital; the mechanism of injury; preexisting medical conditions according to the International Classification of Diseases, 10th revision (ICD-10); diagnoses; surgical and interventional procedures from pre-hospital to inhospital; severity of the injury; and patient outcome and disposition. The diagnosis of the injury is recorded according to the AIS 2005 Update 2008.

\section{DPC Database}

The DPC database contains national administrative claims and discharge data for all inpatients discharged from approximately 1,600 participating hospitals in Japan. Details have been reported elsewhere [25]. Briefly, the database covers all 82 academic hospitals and 91.7\% of all tertiary-care emergency hospitals (244 of 266). In 2018, the number of patients in the database was approximately 8 million. The database includes the following information: diagnoses on admission and complications occurring after admission; classification according to ICD-10; diagnoses data in Japanese; types of surgery; daily doses and records of drugs; records of devices and procedures; and discharge status. A previous study [26] revealed that the validity of diagnostic records in the DPC database is relatively high, with a sensitivity, specificity, and positive predictive value for primary diagnoses of $78.9 \%, 93.2 \%$, and $88.2 \%$, respectively.

\section{PARTICIPANT TIMELINE AND RESEARCH PERIODS}

The timetable and research periods are shown in Table 1 and Fig. 2. After obtaining approval from the Ethics Review Committee, the study will commence, and terminate when the last patient completes follow-up or when 


\begin{tabular}{|c|c|c|c|c|c|c|}
\hline \multirow[b]{3}{*}{ TIMEPOINT } & \multicolumn{6}{|c|}{ STUDY PERIOD } \\
\hline & \multirow{2}{*}{$\begin{array}{l}\text { Enrolment } \\
\text { Admission }\end{array}$} & \multicolumn{5}{|c|}{ Post-injury (month) } \\
\hline & & 2 & 4 & 6 & 12 & 24 \\
\hline \multicolumn{7}{|l|}{ ENROLMENT: } \\
\hline Eligibility screening & $\mathrm{X}$ & & & & & \\
\hline Informed consent & $\mathrm{x}$ & & & & & \\
\hline \multicolumn{7}{|l|}{ ASSESSMENTS: } \\
\hline Patient Social Background & $\mathrm{x}$ & & & & & \\
\hline Japanese Trauma Data Bank Variables & $\mathrm{X}$ & & & & & \\
\hline Death & $\mathrm{X}$ & $\mathrm{X}$ & $\mathrm{X}$ & $\mathrm{X}$ & $\mathrm{X}$ & $\mathrm{X}$ \\
\hline Health-Related Quality of Life & $\mathrm{X}$ & $\mathrm{X}$ & $\mathrm{X}$ & $\mathrm{X}$ & $\mathrm{X}$ & $\mathrm{X}$ \\
\hline Place of Residence & $\mathrm{X}$ & $\mathrm{X}$ & $\mathrm{X}$ & $\mathrm{X}$ & $\mathrm{X}$ & $\mathrm{X}$ \\
\hline Return to Work-Related Outcomes & & $\mathrm{X}$ & $\mathrm{X}$ & $\mathrm{X}$ & $\mathrm{X}$ & $\mathrm{X}$ \\
\hline Frequency of Treatment & & $\mathrm{X}$ & $\mathrm{X}$ & $\mathrm{X}$ & $\mathrm{X}$ & $\mathrm{X}$ \\
\hline Readmission & & $\mathrm{X}$ & $\mathrm{X}$ & $\mathrm{X}$ & $\mathrm{X}$ & $\mathrm{X}$ \\
\hline
\end{tabular}

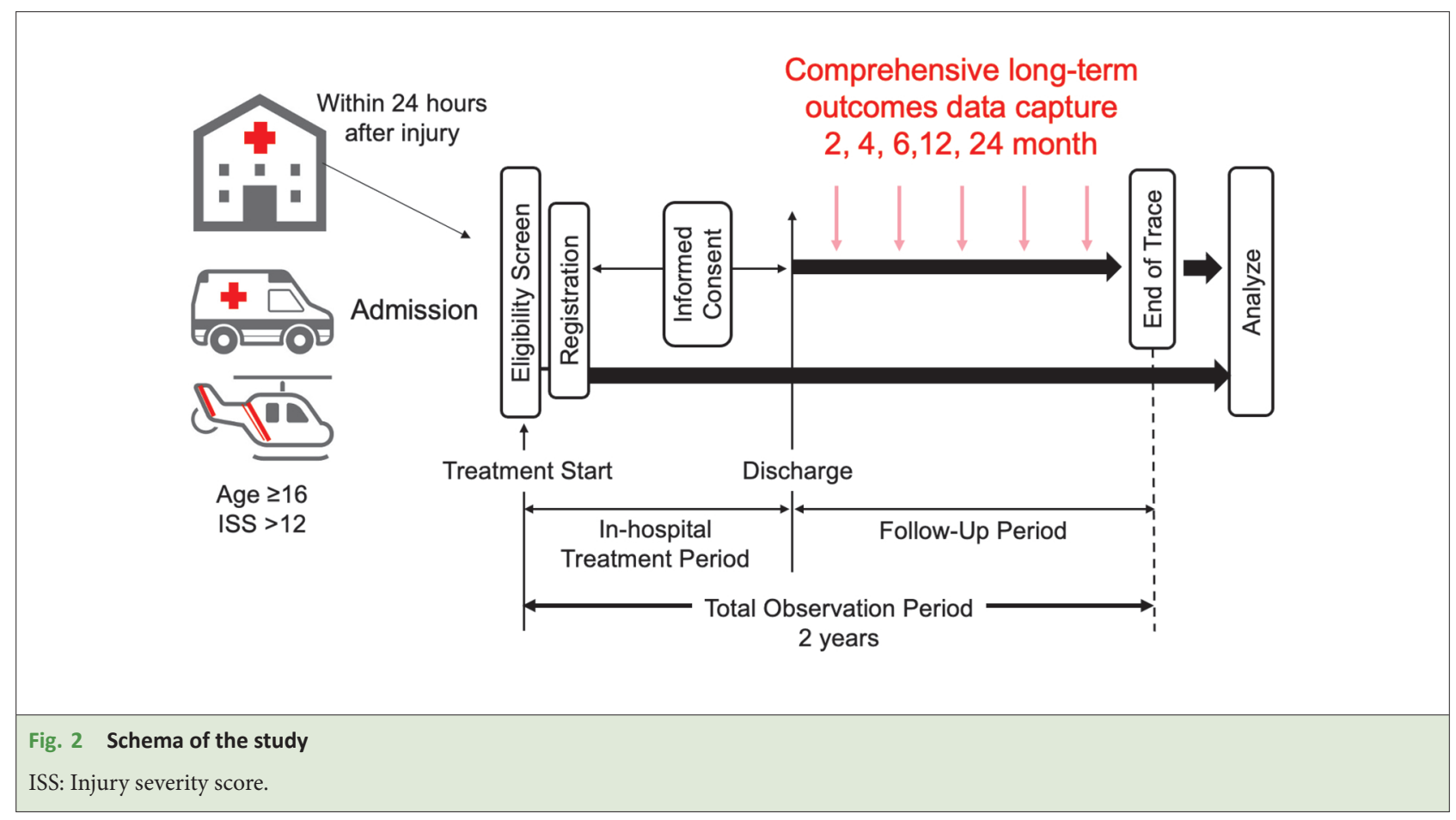

the principal investigator or coinvestigator is unable to contact the patient.

The enrollment period will be 2 years, the observation (follow-up) period will be 2 years after injury, and the total research period will be 4 years from the initiation of the study.

\section{INFORMED CONSENT AND CONFIDENTIALITY}

To receive patient-reported outcomes and track and locate patients, written informed consent will be obtained following approval by the ethics committee. If the patient or their delegate agrees to participate in the study, they will sign a consent form. 


\section{DATA MANAGEMENT}

The principal investigator or research coordinator will create a correspondence table after assigning a new patient an identification number at each facility prior to patient registration.

Participating facilities are allowed to register with JTDB's electronic data capturing system. In addition, the JTDB can freely acquire data registered in its' facilities. The JTDB data will be downloaded at each participating facility, and a patient identification study number will be assigned to the participating facility. Data extraction from the DPC database will be similarly performed at each facility, and a patient identification research number will be assigned within the facility. The clinical information extracted will be sent to the leading research institute with the personal information anonymously processed. Only the patient identification research number will be assigned and combined with the JTDB and DPC data. This information will be integrated at the research center with information from all facilities.

For comprehensive long-term outcome data, information obtained on admission will be entered online with the patient identification research number. After discharge, information will be collected using special questionnaires, and the data collected will be outsourced to a company that has acquired the PrivacyMark (registration number 20000418; PrivacyMark System is a system set up to assess private enterprises that take appropriate measures to protect personal information. Such private enterprises are granted the right to display "PrivacyMark" in the course of their business activities. The System is in compliance with Japan Industrial Standards). With the patients' consent, the outsourcing company will use the patients' personal information to trace them. Thereafter, the company will send anonymized data along with the patient identification research number to the research center.

\section{ANALYSIS}

The study will use quantitative and qualitative methods for the following:

1. For constructing a long-term registry comprising prehospital, in-hospital, and after-hospital data, augmenting the previously existing databases with new data

2. For describing the HRQL, patient trajectory, healthcare use, and social reintegration of seriously injured patients in the first 2 years of injury

3. For assessing the degree of change in the HRQL and the social connection between pre-injury and post-injury 4. For exploring the association between comprehensive long-term outcomes and patient background or trauma care delivery and establish a predictor of long-term trauma outcomes

5. For recommending "tailor-made post-discharge medical care," optimized for each patient to help survivors regain control over their lives.

Continuous variables will be presented as means and standard deviations or medians and quartiles. Categorical variables will be presented as numbers and percentages. Depending on the distribution of each variable, the continuous variable will be analyzed using Student's t-test or the Mann-Whitney U test. Categorical variables will be analyzed using the chi-square test. For descriptive analyses, numbers, graphs, and trends will be described using original scores and key subgroups such as age and severity of illness. Baseline characteristics will not be subjected to significance testing, as suggested in the STROBE guidelines [27]. Patients who can be followed up and those who cannot be followed up may have different characteristics as a long-term follow-up cohort, which may reduce the generalizability of the study results. Therefore, we will compare their background factors to detect potential responder bias.

\section{ANALYSIS OF KEY EVALUATION ITEMS}

Comprehensive Long-Term Patient Outcome Data

The HRQL questionnaires will be provided at the time of discharge and periodically at the 2, 4, 6, 12, and 24 months after injuries. The HQOL questionnaires will be the EQ-5D-5L and SF-12v2. We will describe each subscale of the EQ-5D-5L and SF-12v2, including the visual analog scale, and compare them with the national standard. For the SF-12v2, we will compare the three component scores and the description of each subscale with the national standard. For the EQ-5D-5L, each subscale will be analyzed by converting the subscales into a single index value, followed by converting this value into the QOL value and then comparing the QOL values with the national standard. We will also calculate quality-adjusted life-years. Clinical and socioeconomic factors associated with long-term QOL will be explored using mixed-effects models or generalized estimating equations. Logistic regression models will be used for binary outcomes and linear regression models will be used for continuous variable outcomes. To examine the differences in outcome changes between the subgroups, interaction terms for each subgroup variable and post-injury elapsed time will be entered into the regression model. Pre-injury QOL will be used as a reference; however, recall bias will be considered when comparing QOL values with the 
national standard.

\section{Return to Work}

We will assess whether the patients will be able to return to their job as well as their pre-hospitalization and the post-discharge employment status. We will also use mixed-effects models or generalized estimating equations to explore the clinical and socioeconomic factors associated with reintegration into society. To examine the differences in outcome changes between the subgroups, we will examine each subgroup variable, and the interaction term for post-injury time will be entered into the regression model.

\section{Patient Trajectory}

We will describe the time of survival and discharge periodically for the intervals mentioned above. In addition, we will use mixed-effects models or generalized estimating equations to explore the clinical and socioeconomic factors associated with the patient's current residence. To examine the differences in outcome changes between the subgroups, we will examine each subgroup variable, and the interaction term for post-injury elapsed time will be entered into the regression model.

\section{DISCUSSION}

\section{CONTRIBUTION TO THE FIELD AND SIGNIFICANCE}

This database will be unique as it will contain comprehensive long-term outcomes of patients with trauma focused on patients' backgrounds and patient-reported outcomes. These data are not available in previous trauma databases. This database will accumulate data on socioeconomic status, family structure, place of residence, occupation, pre-injury disability, and mental health status. We will also accumulate information from urban and rural areas, large cities, and medically underdeveloped cities.

The study cohort will be used to create an innovative database. In Japan, a super-aging nation, the age structure of the injured population may differ from that of other countries. Among critically ill patients (injury severity score $\geq 16$ ), the percentage of patients aged $\geq 65$ years was $51 \%$ in the JTDB registry, $35 \%$ in the National Trauma Data Bank registry, and 30\% in the German Trauma Society registry [28-30]. Factors associated with comprehensive long-term outcomes of patients with trauma are also expected to differ from those of other countries. Japan has a universal health care system, which is a compulsory insurance system. All people living in Japan must be covered by public insurance, and all citizens can access standard medical care. Therefore, the type of insurance coverage is not expected to affect longterm prognosis. In contrast, socioeconomic status may affect long-term prognosis. However, based on the National Basic Living Security Act, food, clothing, and shelter are provided for people with financial difficulties to secure the minimum living standard necessary to support their self-sufficiency. Thus, there may be a small detectable relationship between socioeconomic status and long-term outcomes.

By integrating this database with the clinical information obtained and exploring the factors associated with long-term outcomes, we will be able to provide worldleading evidence as a frontrunner of aging society. This evidence will help healthcare providers predict the longterm prognosis of patients with specific conditions and will allow them to make informed health care decisions. It will also assist researchers, clinicians, and policymakers in understanding the impact of specific treatments and trauma care systems on long-term trauma outcomes. This study will provide important evidence from a public health policy perspective, and will recommend strategies for "tailor-made post-discharge medical care," optimized for each patient to help survivors regain control over their lives.

\section{CONFLICT OF INTEREST}

This work will be supported by a JSPS KAKENHI Grant (Grant Number JP19K10499) Grant-in-Aid for Scientific Research(C) received by Dr. Tsuchiya. This funding organization will have no role in the planning of research, analysis, interpretation of results, or report writing.

\section{ACKNOWLEDGMENTS}

This study will be funded by describe above.

The authors would like to thank the multi-center clinical research committee of the Japanese Association for Surgery of Trauma for discussing and developing the protocol.

We would also like to thank Editage (www.editage.com) for English language editing. tion, 2002. pdf. Available from: https:// www.who.int/violence_injury_prevention/ publications/other_injury/injury/en/, 
Accessed 2020 Aug 23.

2. Murray CJL, Vos T, Lozano R, Naghavi M, Flaxman AD, Michaud C, et al. Disabilityadjusted life years (DALYs) for 291 diseases and injuries in 21 regions, 1990-2010: a systematic analysis for the Global Burden of Disease Study 2010. Lancet 2012;380:2197-223.

3. Gore FM, Bloem PJ, Patton GC, Ferguson J, Joseph V, Coffey C, et al. Global burden of disease in young people aged $10-24$ years: a systematic analysis. The Lancet 2011;377: 2093-102.

4. The Ministry of Health, Labour and Welfare: Demographic statistics 2018. https:// www.mhlw.go.jp/toukei/saikin/hw/jinkou/ geppo/nengai18. Accessed 2020 Aug 26.

5. Gabbe BJ, Simpson PM, Cameron PA, Ponsford J, Lyons RA, Collie A, et al. Longterm health status and trajectories of seriously injured patients: A population-based longitudinal study. PLoS Med 2017;14(7).

6. Haider AH, Herrera-Escobar JP, Al Rafai SS, Harlow AF, Apoj M, Nehra D, et al. Factors associated with long-term outcomes after injury: results of the Functional Outcomes and Recovery After Trauma Emergencies (FORTE) multicenter cohort study. Ann Surg 2020;271:1165-73.

7. Gabbe BJ, Simpson PM, Harrison JE, Lyons RA, Ameratunga S, Ponsford J, et al. Return to work and functional outcomes after major trauma: who recovers, when, and how well? Ann Surg 2016;263:623-32.

8. Harcombe H, Langley J, Davie G, Derrett S. Functional status following injury: what recovery pathways do people follow? Injury 2015;46:1275-80.

9. Dutton RP, Stansbury LG, Leone S, Kramer E, Hess JR, Scalea TM. Trauma mortality in mature trauma systems: are we doing better? An analysis of trauma mortality patterns, 1997-2008. J Trauma 2010;69:620-6.

10. Nathens AB. Life after discharge following major injury: expanding our notion of quality in trauma care. Ann Surg 2016;263: 633-5.

11. Berwick DM, Downey AS, Cornett EA. A national trauma care system to achieve zero preventable deaths after injury: recommendations from a National Academies of Sciences, Engineering, and Medicine report. JAMA 2016;316:927-8.

12. Turner GM, Slade A, Retzer A, McMullan C, Kyte D, Belli A, et al. An introduction to patient-reported outcome measures (PROMs) in trauma. J Trauma Acute Care Surg 2019; 86:314-20.

13. The Ministry of Health, Labour and Welfare: The new enhancement stage evaluation of critical care center. https://www.mhlw.go.jp/ content/10800000/000492898.pdf, Accessed 2020 Aug 26.

14. Tsuchiya A, Tsutsumi Y, Yasunaga H. Outcomes after helicopter versus ground emergency medical services for major trauma - propensity score and instrumental variable analyses: a retrospective nationwide cohort study. Scand J Trauma Resusc Emerg Med 2016;24:140.

15. Ware J, Kosinski M, Keller SD. A 12-Item Short-Form Health Survey: construction of scales and preliminary tests of reliability and validity. Med Care 1996;34:220-33.

16. Shiroiwa T, Fukuda T, Ikeda S, Igarashi A, Noto $S$, Saito $S$, et al. Japanese population norms for preference-based measures: EQ-5D-3L, EQ-5D-5L, and SF-6D. Qual Life Res 2016;25:707-19.

17. Fukuhara S, Suzukamo Y. Manual of SF-36v2 Japanese version: iHope International Inc. Kyoto, 2004, 2019.

18. EuroQol Research Foundation. EQ-5D-5L User Guide, 2019. Available from: https:// euroqol.org/publications/user-guides. Accessed 2020 Aug 26.

19. Japan Trauma Care and Research: Japan Trauma Data Bank. https://www.jtcr-jatec.org/ traumabank/dataroom/dataroom.htm. Accessed 2020 Aug 26.

20. Matsuda S, Fujimori K, Fushimi K. Development of casemix based evaluation system in Japan. Asian Pacific Journal of Disease Management 2010;4:55-66.

21. Herdman M, Gudex C, Lloyd A, Janssen
M, Kind P, Parkin D, et al. Development and preliminary testing of the new five-level version of EQ-5D (EQ-5D-5L). Qual Life Res 2011;20:1727-36.

22. Janssen MF, Pickard AS, Golicki D, Gudex C, Niewada M, Scalone L, et al. Measurement properties of the EQ-5D-5L compared to the EQ-5D-3L across eight patient groups: a multi-country study. Qual Life Res 2013;22:1717-27.

23. EuroQol Group. EuroQol-a new facility for the measurement of health-related quality of life. Health Policy 1990;16:199-208.

24. Japan Trauma Data Bank Report 2019 (2014-2018). http://www.jast-hp.org/ trauma/pdf/jtdb2019e.pdf, Accessed 2020 Aug 26.

25. Yasunaga $H$. Real world data in Japan: Chapter II the Diagnosis Procedure Combination Database. Ann Clin Epidemiol 2019;1:76-9.

26. Yamana H, Moriwaki M, Horiguchi $H$, Kodan M, Fushimi K, Yasunaga H. Validity of diagnoses, procedures, and laboratory data in Japanese administrative data. $J$ Epidemiol 2017;27:476-82.

27. Vandenbroucke JP, von Elm E, Altman DG, Gøtzsche PC, Mulrow CD, Pocock SJ, et al. Strengthening the Reporting of Observational Studies in Epidemiology (STROBE): explanation and elaboration. Epidemiology 2007;18:805-35.

28. Japan Trauma Care and Research: Japan Trauma Data Bank Annual Reports 2019. https://www.jtcr-jatec.org/traumabank/ dataroom/dataroom.htm. Accessed 2020 Dec 27.

29. National Trauma Data Bank Reports and Publications 2016. https://www.facs.org/ Quality-Programs/Trauma/TQP/centerprograms/NTDB/docpub. Accessed 2020 Dec 27.

30. Trauma Register DGU General annual Report 2020. http://www.traumaregisterdgu.de/index.php?id=1385\&L=6. Accessed 2020 Dec 27. 